\section{Mit Pregabalin die Radiotherapie unterstützen?}

\author{
Kann mit einem Wirkstoff, der an einen Kalziumkanal bindet, die Schmerz- \\ übertragung gehemmt und die Wirkung einer Radiotherapie bei metastasen- \\ bedingten Knochenschmerzen verstärkt werden?
}

G oldstandard in der Therapie von tumorbedingten Knochenschmerzen ist die Radiotherapie. Doch nur bei etwa jedem fünften Patienten lassen sich die Schmerzen damit vollständig beseitigen. Tierexperimentelle Befunde, wonach Substanzen wie Pregabalin und Gabapentin die Schmerzübertragung hemmen und so die Wirkung der Strahlentherapie unterstützen könnten, wurden nun in einer randomisierten Studie klinisch getestet. 233 Patienten mit radiologisch nachweisbaren Knochenmetastasen erhielten randomisiert eine Radiotherapie (8 Gy in 1 oder 20 Gy in 5 Frak- tionen) plus Pregabalin $(\mathrm{n}=116)$ oder Placebo $(n=117) .35$ Tage lang nahmen die Patienten entweder 2-mal täglich 75 mg Pregabalin oder Placebo ein. Am 1., 8., 15. und 22. Tag wurde die Medikation nach einer Einschätzung der Analgesie angepasst. Primärer Endpunkt war das Therapieansprechen, d.h. die Reduktion der Schmerzen um mindestens 2 Punkte bis Woche 4 bei mindestens stabiler Opioiddosis.

Am häufigsten litten die Teilnehmer unter einem Prostata-, Mamma- oder Lungenkarzinom. Im Pregabalin-Arm erreichten 45 Patienten $(38,8 \%)$ den pri- mären Endpunkt, im Placeboarm 47 (40,2\%; Odds Ratio 1,07; $p=0,816)$. Es gab zwischen den Behandlungsarmen keine signifikanten Unterschiede hinsichtlich Schmerzintensität, Beeinträchtigung von Aktivitäten durch Schmerzen oder Lebensqualität, wohl aber bezüglich Stimmung $(\mathrm{p}=0,031)$ und Durchbruchschmerzen $(p=0,037)$ - jeweils zugunsten der Therapie mit Pregabalin.

Fazit: Die Ergebnisse stützen nicht die Zugabe von Pregabalin zur Radiotherapie bei krebsbedingten Knochenschmerzen. Künftig sollte die Wirkung von Pregabalin bei Knochenschmerzen mit neuropathischer Komponente untersucht werden.

Kathrin von Kieseritzky

Fallon $\mathrm{M}$ et al. Randomized Double-Blind Trial of Pregabalin Versus Placebo in Conjunction With Palliative Radiotherapy for Cancer-Induced Bone Pain. J Clin Oncol. 2016;34(6):550-6.

\title{
Familienintervention bei Krebs?
}

Ist ein Familienmitglied unheilbar an Krebs erkrankt, tut Unterstützung für die gesamte Familie oft not. Nun wurde untersucht, ob eine familienorientierte Therapie die palliative Behandlung von Krebspatienten verbessern kann.

A ngehörige unheilbar kranker Patienten können Hilfe und Unterstützung oft gut gebrauchen - in der Pflege und im täglichen Umgang mit der Krankheit ebenso wie in der Trauer, wenn der Sterbefall eingetreten ist. Doch evidenzbasierte Modelle für eine familienzentrierte Betreuung von Krebspatienten fehlen bislang. In der aktuellen Studie wurden Patienten mit fortge-

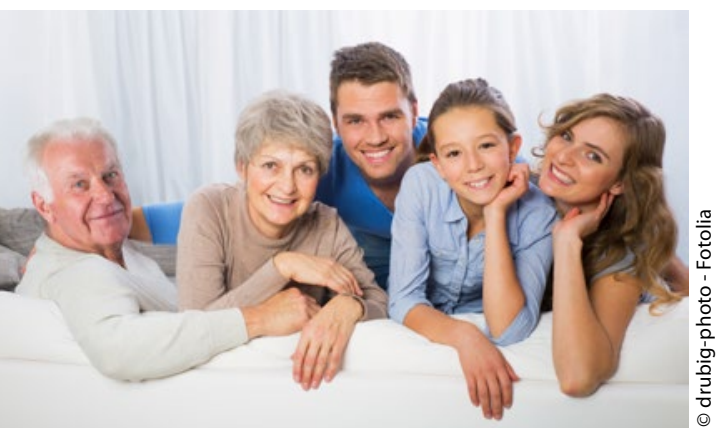

10 Stunden Familienintervention erwiesen sich als sehr positiv.

schrittener maligner Erkrankung und ihre Angehörigen mithilfe des Family Relationships Index gescreent. Von 1.488 Patienten bzw. Angehörigen kristallisierten sich 620 (42\%) mit Konfliktpotenzial in der Familie als Teilnehmer heraus. Sie stammten aus insgesamt 170 Familien, die jeweils einer der folgenden 3 Kategorien zugeordnet wurden: „wenig Kommunikation“, „wenig Beteiligtsein“ oder „viele Konflikte“. Die Familien wurden randomisiert einem von 3 Behandlungsarmen zugeteilt: Standardpflege oder 6 oder 10 Sitzungen Familienintervention. Als Messinstrumente dienten das Complicated Grief Inventory-Abbreviated (CGI) und das Beck Depression Inventory-II (BDI-II).

Im CGI zeigte sich ein signifikanter positiver Effekt durch die Familienintervention $(p=0,032)$. Zudem fand sich eine Interaktion zwischen der Therapie und dem Familientyp $(\mathrm{p}<0,001)$. Bei den Gruppen „wenig Kommunikation“ bzw. „viele Konflikte" führten 10 Sitzungen Familienin- tervention im Vergleich zur Gruppe „wenig-Beteiligtsein“ zu besseren Ergebnissen im CGI ( $p<0,001$ bzw. 0,039).

Vor allem Familien mit wenig Kommunikation konnte durch die Familienintervention in den ersten 6 Monaten bei der Trauer um einen verstorbenen Angehörigen geholfen werden $(p=0,0104)$. Der positive Effekt war auch nach 13 Monaten der Trauer deutlich $(p=0,017)$. Auch in Familien mit vielen Konflikten wurde ein solcher Effekt gefunden, der aber nicht signifikant war.

Im Standardpflege-Arm wiesen 15,5\% der Angehörigen nach 13 Monaten eine verlängerte und gestörte Trauer auf, im Arm mit 10 Sitzungen Familienintervention nur 3,3\% ( $\mathrm{p}=0,048)$.

Fazit: Eine familienfokussierte Therapie kann während der palliativen Therapie unheilbar krebskranker Patienten sowie in der Trauerzeit nach deren Tod bei Familien mit hohem Konflikt- und Komplikationspotenzial so unterstützend wirken, dass das Risiko für anhaltende Trauerstörungen ebenso sinkt wie deren Intensität.

Kathrin von Kieseritzky

Kissane DW et al. Randomized Controlled Trial of Family Therapy in Advanced Cancer Continued Into Bereavement. J Clin Oncol. 2016, 34(16):1921-7. 